Planetary Systems in the Universe - Observation, Formation and Evolution

Proceedings IAU Symposium No. 202, (C2004 IAU

Alan Penny, Pawel Artymowicz, Anne-Marie Lagrange, 8 Sara Russell, eds.

\title{
Observations of YSO Circumstellar Discs
}

\author{
D. Ward-Thompson
}

Dept of Physics \& Astronomy, Cardiff University, PO Box 913, Cardiff, $U K$

P. André

CEA,Service d'Astrophysique, C.E. Saclay, Gif-sur-Yvette, France

O. P. Lay

Jet Propulsion Lab, 4800 Oak Grove Drive, Pasadena, California, USA

\begin{abstract}
We present the results of short baseline interferometry observations at submillimetre wavelengths, using a two-element interferometer comprising the JCMT/CSO, of circumstellar discs around young YSOs. We model data for the Class 0 protostar IRAS03282 and the Class I protostar L1709B with discs and compare them with previously published results. We find evidence for the ratio of the disc to envelope mass to increase as the objects evolve.
\end{abstract}

\section{Introduction}

Circumstellar discs now appear to be an ubiquitous phenomenon around newlyformed stars. One of the most direct ways to study discs is to use submm interferometry. For optically thin emission, the submm flux density is proportional to the mass of the circumstellar disc. A number of young stars have now been studied with submm interferometers, including that involving the linking of the JCMT \& CSO to perform short-baseline interferometry (SBI).

\section{IRAS 03282}

IRAS 03282 is a Class 0 YSO (André, Ward-Thompson \& Barsony 1993; 2000), which is in a very early proto-stellar phase. Figure 1(a) shows a plot of flux density against projected baseline for our IRAS 03282 data. We note that the data are consistent with a constant flux density of $\sim 0.4 \mathrm{Jy}$ from $\sim 100-200 \mathrm{k} \lambda$, corresponding to angular resolution from 1.25-2.5 arcsec. At an assumed distance of 350 pc (Bachiller \& Cernicharo 1986) this corresponds to scales of 440-880AU. We interpret this as an unresolved component, presumably a disc, around this source. The outer radius of this disc is thus $<220 \mathrm{AU}$.

Using typical assumptions (e.g. André et al. 2000) of optically thin dust emission at $\mathrm{T}=30 \mathrm{~K}$ and mass opacity at this frequency of $\kappa=0.0015 \mathrm{~m}^{2} \mathrm{~kg}^{-1}$, a flux density of $0.4 \mathrm{Jy}$ at a distance of $350 \mathrm{pc}$ corresponds to a mass of $\sim 0.1 \mathrm{M}_{\odot}$. The single-dish submm flux density at the same wavelength is $1.4 \mathrm{Jy}$ in a 13 arcsec 
IRAS 03282

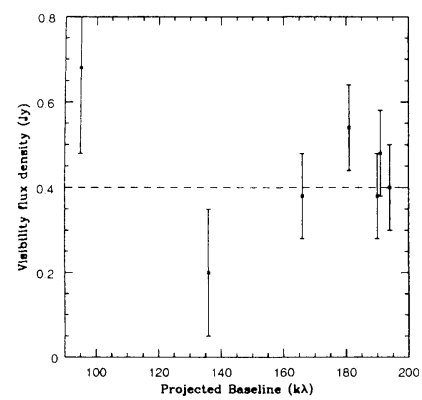

L1709B

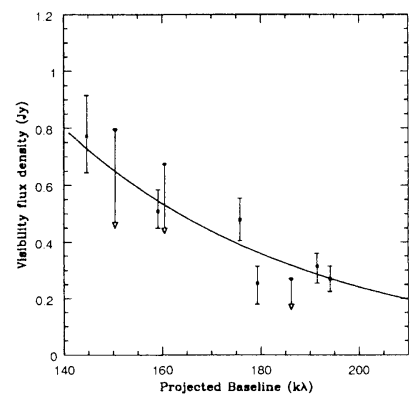

Figure 1. Plots of flux density versus projected baseline. (a) IRAS03282: Note that there is no real trend in the data, and a constant flux density of $0.4 \mathrm{Jy}$ is consistent with the data. This corresponds to an unresolved disc of $0.1 \mathrm{M}_{\odot}$ of radius $<220 \mathrm{AU}$. (b) L1709B: The solid line is a gaussian fit to the data with FWHM $~ 60$ AU.

beam (Barsony et al 1998). This corresponds to a scale of $4500 \mathrm{AU}$. This is clearly emission from the envelope around this Class 0 protostar (see: Barsony et al 1998). Thus the ratio of the flux in the interferometer measurements to the single dish flux, which approximates to the ratio between the disc emission and the envelope emission, is $\sim 30 \%$.

\section{L1709B}

L1709B is a Class I protostar at a distance of $140 \mathrm{pc}$, and is among the stronger mm sources in the $\rho$ Oph dark cloud star-forming region (André \& Montmerle 1994). Figure 1(b) shows a plot of flux density against projected baseline for our L1709B data. In this case we see that the data are not consistent with constant flux density on all baselines, so we have at least partially resolved the emission. We make the assumption that the emission is arising from an optically thin disc, which has a gaussian radial density profile. We plot a gaussian fit to the data and find a FWHM flux density of $0.4 \mathrm{Jy}$ on a scale of $175 \mathrm{k} \lambda$, corresponding to a FWHM disc size of $\sim 0.45$ arcsec, or $\sim 60 \mathrm{AU}$ at the distance of L1709B. The single dish flux density of L1709B is 1.5Jy (André et al 1993), and we attribute this to the extended envelope emission around this source. The ratio of interferometer FWHM flux to single dish flux, under typical assumptions, is therefore $\sim 30 \%$. We have also observed three other sources but can only place limits on their fluxes.

\section{Comparison with previous SBI data}

We can compare our results with those of other workers using the same interferometer on similar sources. These are listed in Table 1, along with our new detections and upper limits. The SBI flux corresponds to the disc, and the single-dish (14") JCMT flux is emission from the disc-plus-envelope. Thus we 


\begin{tabular}{llccccc} 
Source & Cloud & Class & \multicolumn{2}{c}{ SBI } & JCMT & Ratio \\
Name & Name & & $\begin{array}{c}\text { Flux } \\
(\mathrm{Jy})\end{array}$ & $\begin{array}{c}\text { Scale } \\
(\mathrm{k} \lambda)\end{array}$ & $\begin{array}{c}\text { Flux } \\
(\mathrm{Jy})\end{array}$ & \\
& & & & & & \\
L1448mm & Perseus & 0 & $0.12^{4}$ & $100-200$ & $1.9^{6}$ & $6 \%$ \\
SMM3 & Serpens & 0 & $0.2^{4}$ & $100-200$ & $1.8^{7}$ & $11 \%$ \\
VLA1623 & $\rho$ Oph & 0 & $0.5^{3}$ & $140-200$ & $4^{8,9}$ & $12 \%$ \\
NGC1333IRAS2A & Perseus & 0 & $0.4^{4}$ & $100-200$ & $3.1^{6}$ & $13 \%$ \\
FIRS1 & Serpens & 0 & $1.3^{4}$ & $100-200$ & $7^{7}$ & $19 \%$ \\
IRAS03282 & Perseus & 0 & $0.4^{1}$ & $100-200$ & $1.4^{5}$ & $29 \%$ \\
LFAM1 & $\rho$ Oph & 0 & $\leq 1^{1}$ & $140-200$ & $1.8^{1}$ & $\leq 55 \%$ \\
IRS53 & Serpens & I & $0.15^{4}$ & $100-200$ & $1.7^{7}$ & $9 \%$ \\
SVS13A & Perseus & I & $0.7^{4}$ & $100-200$ & $2.7^{6}$ & $26 \%$ \\
SVS20 & Perseus & I & $0.3^{4}$ & $100-200$ & $1.1^{6}$ & $27 \%$ \\
L1709B & $\rho$ Oph & I & $0.4^{1}$ & 175 & $1.5^{1}$ & $27 \%$ \\
L1551-IRS5 & Taurus & I & $2.2^{2}$ & 50 & $7.8^{6}$ & $28 \%$ \\
El24 & $\rho$ Oph & II & $\leq 0.5^{1}$ & $140-200$ & $0.9^{1}$ & $\leq 60 \%$ \\
DoAr25 & $\rho$ Oph & II & $\leq 1^{1}$ & $140-200$ & $0.7^{1}$ & $\leq 100 \%$ \\
HLTau & Taurus & II & $2.6^{2}$ & 50 & $2.6^{6}$ & $100 \%$
\end{tabular}

Table 1. Comparison with previous SBI data. Notes: ${ }^{1}$ This work; ${ }^{2}$ Lay et al $1994 ;{ }^{3}$ Pudritz et al $1996 ;{ }^{4}$ Brown et al $2000 ;{ }^{5}$ Barsony et al $1998 ;{ }^{6}$ Chandler \& Richer 2000; ${ }^{7}$ Casali et al $1993 ;{ }^{8}$ André et al $1993 ;{ }^{9}$ Ward-Thompson 1993.

can see a possible trend emerging from this table. All except one of the Class 0 sources with detections have SBI to single dish flux ratios of $<20 \%$. All except one of the Class I sources with detections have SBI to single dish flux ratios of $20-30 \%$. The one detected Class II source has an SBI to single dish flux ratio of $100 \%$. We interpret this as a possible evolutionary trend from Class $0 \rightarrow I \rightarrow I I$, caused by the ratio of the disc to envelope mass increasing with time as a result of the envelope matter accreting onto the disc.

\section{References}

André \& Montmerle 1994, ApJ, 420, 837

André, Ward-Thompson \& Barsony 1993, ApJ, 406, 122

André, Ward-Thompson \& Barsony 2000, 'Protostars \& Planets IV', 59

Bachiller \& Cernicharo 1986, A\&A, 168, 262

Barsony et al 1998 ApJ, 509, 733

Brown et al 2000, MNRAS, in press

Casali et al 1993, A\&A, 275, 195

Chandler \& Richer 2000, ApJ, 530, 851

Lay et al 1994, ApJ, 434, L75

Pudritz et al 1996, ApJ, 470, L123

Ward-Thompson 1993, MNRAS, 265, 493 